\title{
Papers
}

\section{Maternal and paternal age at delivery, birth order, and risk of childhood onset type 1 diabetes: population based cohort study Topic: 24;202;72}

Lars C Stene, Per Magnus, Rolv T Lie, Oddmund Søvik, Geir Joner, and the Norwegian Childhood Diabetes Study Group

\begin{abstract}
Objective To estimate the associations of maternal and paternal age at delivery and of birth order with the risk of childhood onset type 1 diabetes.

Design Cohort study by record linkage of the medical birth registry and the national childhood diabetes registry in Norway.

Setting Norway.

Subjects All live births in Norway between 1974 and 1998 (1.4 million people) were followed for a maximum of 15 years, contributing 8.2 million person years of observation during 1989-98. 1824 cases of type 1 diabetes diagnosed between 1989 and 1998 were identified.

Main outcome measures Incidence of type 1 diabetes.

Results There was no association between maternal age at delivery and type 1 diabetes among firstborn children, but among fourthborn children there was a $43.2 \%$ increase in incidence of diabetes for each five year increase in maternal age $(95 \%$ confidence interval $6.4 \%$ to $92.6 \%$ ). Each increase in birth order was associated with a $17.9 \%$ reduction in incidence (3.2\% to $30.4 \%)$ when maternal age was $20-24$ years, but the association was weaker when maternal age was 30 years or more. Paternal age was not associated with type 1 diabetes after maternal age was adjusted for.
\end{abstract}

Conclusions Intrauterine factors and early life environment may influence the risk of type 1 diabetes. The relation of maternal age and birth order to risk of type 1 diabetes is complex.

\section{Introduction}

Type 1 diabetes mellitus is caused by immune mediated destruction of the pancreatic $\beta$ cells. Both genetic and non-genetic factors are involved in the pathogenesis, but the factors initiating the destructive process are largely unknown. Environmental risk factors may have a role early in life, possibly in utero. ${ }^{1}$

Several studies have investigated the relation between maternal age at delivery or birth order and risk of childhood onset type 1 diabetes. ${ }^{2-13}$ Many of these studies were relatively small, used census data as controls or siblings as controls, and inappropriately analysed the data as if they arose from a cohort study. ${ }^{2} 71314$ Some studies found a weak increase in risk of type 1 diabetes in children born to older mothers, although others found no significant association.

Studies of birth order have given particularly inconsistent results. Maternal age and birth order are correlated, and the inconsistent findings may be explained by differences in adjustment for maternal age. Furthermore, interaction between maternal age and birth order may exist. Results from studies of association between paternal age at delivery and risk of type 1 diabetes have also been inconsistent. ${ }^{57812}$ The objective of this study was to estimate the associations of maternal and paternal age at delivery and of birth order with the incidence of type 1 diabetes and the interactions between these variables.

\section{Subjects and methods}

In Norway, all newly diagnosed cases of type 1 diabetes in children under 15 years old have been prospectively registered in the national childhood diabetes registry since 1 January $1989 .{ }^{15}$ We designed a cohort study linking records in the medical birth registry with those in the national childhood diabetes registry through the unique personal identification number assigned to all residents of Norway. We linked 1824 of the 1863 cases of type 1 diabetes diagnosed between 1 January 1989 and 31 December 1998. The study was approved by the regional ethics committee and the National Data Inspectorate.

All live births in Norway between 1974 and 1998 contributed time under observation from birth to diagnosis of type 1 diabetes (from 1989 to 1998), age 15 years, death in the first year of life, or 31 December 1998, whichever occurred first. Since registration of type 1 diabetes started in 1989, time under observation was counted only from 1 January 1989 for those born before this date. This means that even if some children developed type 1 diabetes before 1989, it would not influence the results of this study. We did not have information on deaths occurring between age 1 and 15 years, but these are rare and would not influence our results.

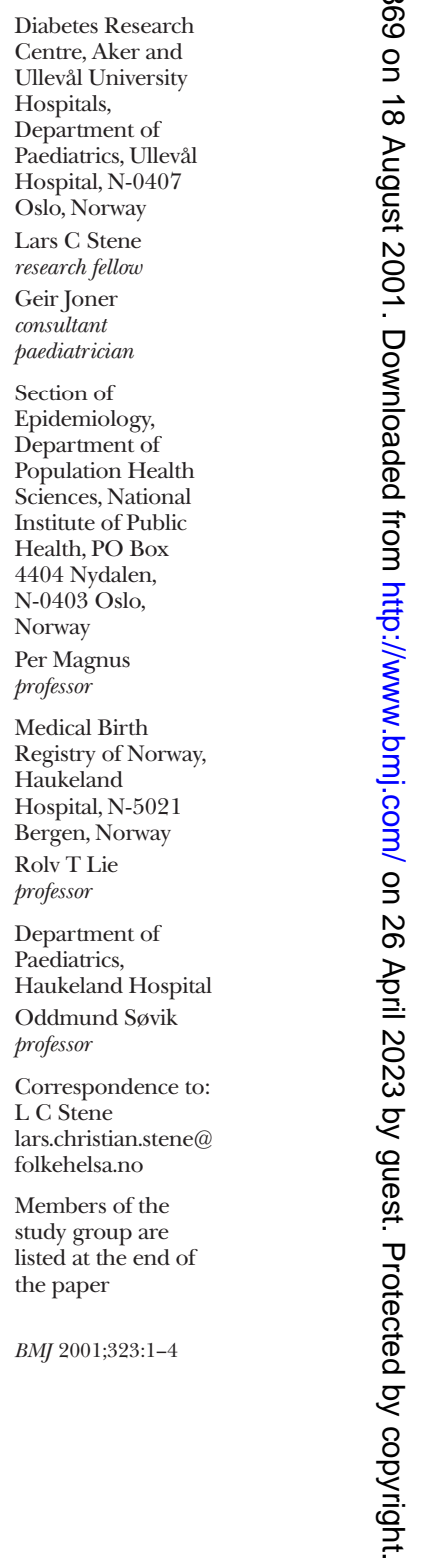


We calculated maternal and paternal age as the difference between the birth year of the index child and the birth year of the mother and father, respectively. The child's birth order was inferred from the number of previous births (including stillbirths) reported by the mother at the time of birth of the index child (1. $9 \%$ of mothers who had at least one previous birth reported at least one previous stillbirth). We excluded $0.1 \%$ of the cohort because of missing data on birth order, including 12 who developed diabetes. In analyses of paternal age, we excluded $7.6 \%$ with missing data, including 118 who developed diabetes. Parental age and birth order were divided into five categories (see table 1).

We calculated the number of incident cases and person years under observation in each exposure category using Datab in the Epicure package, version $1.8 \mathrm{w}^{16}{ }^{16} \mathrm{We}$ calculated incidence as the number of incident cases divided by the person years under observation in each category. We estimated rate ratios with 95\% confidence intervals by Poisson regression analyses. Exposure variables were also entered as continuous variables with five levels corresponding to the mean in each category. ${ }^{16}$ We calculated percentage differences in incidence associated with given changes in exposure by subtracting one from the rate ratios and multiplying by 100 . We used the likelihood ratio test for exposures entered as continuous variables to test for trend.

We included calendar period of birth in five year categories and age group in three year categories to adjust for possible period effects. We also entered maternal diabetes mellitus diagnosed before or during the index pregnancy, pre-eclampsia, bleeding during pregnancy, caesarean delivery, sex, and birth weight (as a continuous variable $)^{17}$ to evaluate confounding. To assess whether the associations of parental age and birth order with type 1 diabetes were the same for different levels of other exposures, age groups, sex, and calendar periods, we inspected the results of stratified

Table 1 Maternal and paternal age at delivery, birth order, and rate ratio of childhood onset type 1 diabetes

\begin{tabular}{|c|c|c|c|c|}
\hline & \multirow[b]{2}{*}{ No with diabetes } & \multirow[b]{2}{*}{ Person years } & \multicolumn{2}{|c|}{ Rate ratio $(95 \% \mathrm{Cl})$} \\
\hline & & & Unadjusted & Adjusted ${ }^{*}$ \\
\hline \multicolumn{5}{|c|}{ Maternal age (years) } \\
\hline$<20$ & 99 & 451574 & 1.0 & 1.0 \\
\hline $20-24$ & 468 & 2219415 & $0.96(0.77$ to 1.20$)$ & 1.00 (0.80 to 1.24$)$ \\
\hline $25-29$ & 659 & 2982226 & 1.01 (0.82 to 1.25$)$ & 1.09 (0.88 to 1.35$)$ \\
\hline $30-34$ & 414 & 1788072 & $1.06(0.85$ to 1.32$)$ & 1.18 (0.94 to 1.46$)$ \\
\hline$\geqslant 35$ & 172 & 672566 & 1.17 (0.91 to 1.49$)$ & 1.34 (1.04 to 1.71$)$ \\
\hline Test for trend & & & $P=0.06$ & $P<0.001$ \\
\hline \multicolumn{5}{|l|}{ Birth order } \\
\hline 1st & 772 & 3457974 & 1.0 & 1.0 \\
\hline 2nd & 648 & 2886296 & 1.01 (0.91 to 1.12$)$ & 1.01 (0.91 to 1.12$)$ \\
\hline $3 \mathrm{rd}$ & 279 & 1291460 & 0.97 (0.84 to 1.11$)$ & 0.98 (0.85 to 1.12$)$ \\
\hline 4th & 79 & 340145 & $1.04(0.83$ to 1.31$)$ & 1.05 (0.83 to 1.32$)$ \\
\hline$\geqslant 5$ th & 34 & 137978 & $1.10(0.78$ to 1.56$)$ & 1.10 (0.78 to 1.55$)$ \\
\hline Test for trend & & & $P=0.78$ & $\mathrm{P}=0.70$ \\
\hline \multicolumn{5}{|c|}{ Paternal age (years) } \\
\hline$<25$ & 298 & 1318112 & 1.0 & 1.0 \\
\hline $25-29$ & 572 & 2696972 & 0.94 (0.82 to 1.08$)$ & 0.96 (0.83 to 1.10$)$ \\
\hline $30-34$ & 517 & 2167845 & 1.06 (0.91 to 1.22$)$ & $1.11(0.96$ to 1.28$)$ \\
\hline $35-39$ & 221 & 953271 & 1.03 (0.86 to 1.22$)$ & 1.11 (0.93 to 1.32$)$ \\
\hline$\geqslant 40$ & 98 & 410621 & 1.06 (0.84 to 1.33$)$ & 1.16 (0.92 to 1.46$)$ \\
\hline Test for trend & & & $P=0.33$ & $P=0.02$ \\
\hline
\end{tabular}

${ }^{\star}$ Adjusted for age group and year of birth.

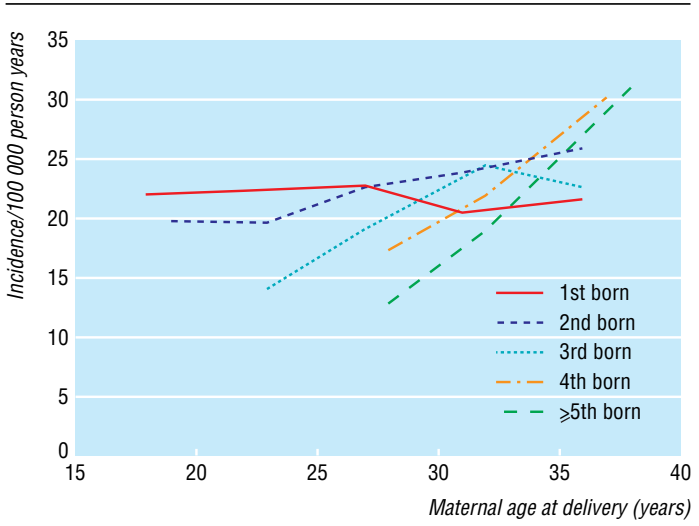

Maternal age at delivery and incidence of childhood onset type 1 diabetes by birth order. Data points are crude incidences in the maternal age groups $<20,20-24,25-29,30-34$, and $\geqslant 35$ years, with the points placed at the mean maternal age within each category. There were no diabetic people with certain rare combinations of maternal age and birth order (birth order $\geqslant 3$ with maternal age $<20$ and birth order $\geqslant 4$ with maternal age $<25$ )

analyses and tested the significance of the respective interaction terms.

\section{Results}

A total of 1382602 individuals contributed 8166731 person years under observation between 1989 and 1998. The mean time from birth to censoring or type 1 diabetes was $10.2(\mathrm{SD}=5.0)$ years, and the mean time under observation after 1 January 1989 was 5.9 (3.3) years. The mean age at diagnosis among the 1824 who developed type 1 diabetes was 8.6 (3.7) years.

We found a weak crude association between maternal age at delivery and incidence of type 1 diabetes. This became somewhat stronger and significant after year of birth and age group were adjusted for (table 1). There were no significant crude associations between birth order and incidence of type 1 diabetes (table 1). When both maternal age and birth order were included in the model, the rate ratios for maternal age were hardly changed but birth order became weakly negatively associated with type 1 diabetes (test for trend; $\mathrm{P}=0.06$ ). However, after stratification, an interaction between maternal age at delivery and birth order appeared.

We found no association between maternal age at delivery and incidence of type 1 diabetes among firstborn children, but among second or later born children there was a positive association. The strength of the association increased with birth order (figure). In a regression model with maternal age and birth order entered as continuous variables, their multiplicative interaction was highly significant $(\mathrm{P}=0.004)$. Table 2 shows the estimated change in incidence for each five year increase in maternal age from regression models stratified by birth order. For instance, among fourthborn children each five year increase in maternal age was associated with a $43.2 \%$ increase $(95 \%$ confidence interval $6.4 \%$ to $92.6 \%$ ) in incidence of type 1 diabetes.

Compared with firstborn children, the incidence of type 1 diabetes was lower for second or later children when maternal age was low (figure). For instance, among children born to mothers aged 20-24 years at 
Table 2 Estimated associations of maternal age at delivery and birth order with incidence of type 1 diabetes from stratified Poisson regression models with adjustment for age group and calendar period of birth. All values are percentages

Change in incidence $(95 \% \mathrm{Cl})$

\begin{tabular}{ll}
\hline Birth order* & \\
\hline 1st & $4.0(-4.4$ to 13.1$)$ \\
\hline 2nd & $16.7(6.0$ to 28.5$)$ \\
\hline 3rd & $21.8(4.5$ to 42.0$)$ \\
\hline 4th & $43.2(6.4$ to 92.6$)$ \\
\hline th & $51.3(-7.6$ to 148.0$)$ \\
\hline
\end{tabular}

Maternal age (years) $\dagger$

\begin{tabular}{lc}
\hline$<20$ & $-14.4(-61.8$ to 91.4$)$ \\
\hline $20-24$ & $-17.9(-30.4$ to -3.2$)$ \\
\hline $25-29$ & $-9.6(-18.0$ to -0.4$)$ \\
\hline $30-34$ & $-2.1(-11.0$ to 7.7$)$ \\
\hline$\geqslant 35$ & $4.8(-5.5$ to 16.2$)$
\end{tabular}

${ }^{\star}$ Change per five year increase in maternal age.

†Change per increase in birth order.

delivery, each increase in birth order was associated with a $17.9 \%$ reduction in the incidence of type 1 diabetes $(3.2 \%$ to $30.4 \%)$. The association was weaker or non-existent when maternal age at delivery was 30 years or more (table 2 ).

There was a weak association between incidence of type 1 diabetes and paternal age at delivery. This was significant after adjustment for calendar period of birth and age group (table 1). The same patterns that were found for maternal age were also found for paternal age. However, the strength of the associations and the interaction with birth order were weaker than those for maternal age and the $\mathrm{P}$ values were larger (data not shown). After the effects of maternal age and birth order were adjusted for, there was no significant association between paternal age and type 1 diabetes. The effects of maternal age and birth order, however, were essentially unchanged after adjustment for paternal age.

The incidence of type 1 diabetes showed a small non-significant decrease during the study and was slightly higher among boys than girls. We previously found a weak but significant positive association between birth weight and incidence of type 1 diabetes, ${ }^{17}$ but in this study there was no association between the other potential confounding factors evaluated and incidence of type 1 diabetes. All main results were essentially independent of potential confounders evaluated. The associations were similar for each sex, year of birth, and age group, although the main effect of maternal age was slightly stronger in the earlier birth periods than later periods $(\mathrm{P}=0.03$ for interaction between maternal age and birth period in model with only maternal age and period and in model including age group, birth order, and interaction term between maternal age and birth order).

\section{Discussion}

We found that the association between maternal age and childhood onset type 1 diabetes increased with rising birth order. Furthermore, any association between paternal age and type 1 diabetes was secondary to maternal age at delivery. The advantages of this study were the large sample size and the fact that the data were based on computerised registries with nearly complete coverage. We cannot, however, exclude confounding by unmeasured factors such as socioeconomic status of the children's families, maternal smoking during pregnancy, or whether delayed or early childbearing and number of births were intentional.

\section{Other studies}

A previous study which tested for an interaction between maternal age and birth order found no significant interaction. ${ }^{13}$ One problem with this study was that the authors used cohort analysis of data arising from a case-control study with siblings as controls. ${ }^{13}$ This probably biased their results. ${ }^{14}$ Furthermore, results from families with type 1 diabetes cannot readily be generalised to the total population. On the other hand, we cannot exclude the possibility that the effects of maternal age and birth order on risk of type 1 diabetes are different in different populations.

Several studies have found a significant crude association between increased maternal age and increased risk of type 1 diabetes..$^{2-5} 7101113$ Some of these studies used national reference data as controls ${ }^{2}$ or used siblings as controls. ${ }^{3}{ }^{13}$ In some studies, the association was reported to disappear after birth order or other potential confounders were adjusted for. ${ }^{718}$ Other studies, often with relatively small sample sizes, did not find any significant crude association between maternal age at delivery and type 1 diabetes. ${ }^{9}$

\section{Birth order and paternal age}

Associations of birth order and paternal age with risk of type 1 diabetes have been particularly inconsistent in previous studies. Both significantly higher risk $^{57}$ and lower risk ${ }^{11}$ among firstborn children compared with second or later born children have been found, and there was evidence of heterogeneity between centres in two of these studies. ${ }^{511}$ Some studies have not found any significant association between birth order and type 1 diabetes. ${ }^{9} 10^{12}$ Paternal age at delivery has been negatively associated ${ }^{7}$ and positively associated with risk of type 1 diabetes. ${ }^{5}$ One study found a U shaped relation, ${ }^{8}$ but others found no significant association. ${ }^{12}$ Our results indicate that these inconsistencies may be due to small sample sizes, no adjustment for relevant confounders, and lack of stratification by maternal age and birth order.

\section{Possible explanations for the effect}

Maternal age and parity are associated with various sociodemographic and biological factors. Delayed childbearing is associated with longer maternal education, complications in pregnancy, lower birth weight, fetal loss, and perinatal mortality. ${ }^{19}{ }^{20}$ Some of these associations depend on maternal parity. ${ }^{19}$ Pregnancies at older maternal ages are probably a mix of intentional and non-intentional pregnancies. Parous women who give birth at older ages may have had short or long intervals between previous pregnancies and may have had varying number of previous abortions or stillbirths. ${ }^{20}$ Increasing age of the mother may be a marker for accumulated exposures such as infections or environmental toxins. Fetomaternal immune responses may also change with each pregnancy, ${ }^{21}$ and this could partly explain our results.

Maternal age and birth order are also likely to influence a child's environment in early life. For instance, feeding practices, neonatal care, and expo- 


\section{What is already known on this topic}

Maternal age at birth is positively associated with risk of childhood onset type 1 diabetes

Studies of the effect of birth order on risk of type 1 diabetes have given inconsistent results

\section{What does this study add?}

In a national cohort, risk of diabetes in firstborn children was not associated with maternal age

Increasing maternal age was a risk factor in children born second or later

The strength of the association increased with increasing birth order

sure to infections may differ depending on the age of the mother and the number of siblings. McKinney et al have found some evidence that early attendance at daycare centres, as a measure of childhood infections, is associated with lower risk of type 1 diabetes. ${ }^{22}$ However, conflicting evidence from other studies exists. ${ }^{23}$

We were unable to determine whether spacing between pregnancies is relevant. In addition, we had no data on whether the number of previous pregnancies, including those that ended in fetal loss, affected the risk of type 1 diabetes in children.

In conclusion, we found a significant interaction between maternal age at delivery and birth order in relation to incidence of type 1 diabetes. This indicates that the relation between maternal age, birth order, and risk of type 1 diabetes is more complex than previously thought.

We thank the staff at the medical birth registry for their help

Members of the Norwegian Childhood Diabetes Study Group were Henning Aabech, Fredrikstad; Helge Vogt, Nordbyhagen; Knut Dahl-Joergensen, Oslo; Hans-Jacob Bangstad, Oslo; Geir Joner, Oslo; Kolbeinn Gudmundsson, Oslo; Olav Flesvig, Elverum; Halvor Baevre, Lillehammer; Ola Talleraas, Lillehammer; Kjell Stensvold, Drammen; Bjørn Halvorsen, Toensberg; Kristin Hodnekvam, Porsgrunn; Ole Kr Danielsen, Arendal; Jorunn Ulriksen, Kristiansand; Geir Stangeland, Kristiansand; John Bland, Stavanger; Dag Roness, Haugesund; Oddmund Soevik, Bergen; Per Helge Kvistad, Foerde; Steinar Spangen, Aalesund; Per Eirik Haereid, Trondheim; Sigurd Boersting, Levanger; Dag Veimo, Bodoe; Harald Dramsdahl, Harstad; and Kersti Thodenius, Hammerfest.

Contributors: OS and GJ had the idea for the study, and GJ was the principal investigator. RTL and PM provided advice in presentation and interpretation of the results. LCS formulated the analysis strategy, carried out all data analyses, and wrote the paper. All authors helped interpret the findings and commented on earlier drafts of the manuscript. All members of the
Norwegian Childhood Diabetes Study Group registered newly diagnosed cases of childhood onset type 1 diabetes. LCS and GJ are guarantors.

Funding: LCS and GJ were supported by a grant from the Norwegian Foundation for Health and Rehabilitation (grant no 1997/156) and a grant from the Norwegian Diabetes Association. Funding was also kindly provided by TINE Norwegian Dairies and Novo Nordisk Pharma A/S

Competing interests: None declared.

1 Leslie DG, Elliott RB. Early environmental events as a cause of IDDM. Diabetes 1994:43:843-50.

2 Flood TM, Brink SJ, Gleason RE. Increased incidence of type I diabetes in children of older mothers. Diabetes Care 1982;6:571-3.

3 Wagener DK, LaPorte RE, Orchard TJ, Cavender D, Kuller LH, Drash AI The Pittsburgh diabetes mellitus study 3: an increased prevalence with older maternal age. Diabetologia 1983;25:82-5.

4 Dahlquist G, Källén B. Maternal-child blood group incompatibility and other perinatal events increase the risk for early-onset type 1 (insulin-dependent) diabetes mellitus. Diabetologia 1992;35:671-5.

5 Patterson CC, Carson DJ, Hadden DR, Waugh N, Cole SK. A case-control investigation of perinatal risk factors for childhood IDDM in Northern Ireland and Scotland. Diabetes Care 1994;17:376-81.

6 Soltesz G, Jeges S, Dahlquist G. Non-genetic risk determinants for type 1 (insulin-dependent) diabetes mellitus in childhood. Acta Paediatr 1994:83:730-5.

7 Wadsworth EJK, Shield JPH, Hunt LP, Baum JD. A case-control study of environmental factors associated with diabetes in the under $5 \mathrm{~s}$. Diabetic Med 1997;14:390-6.

8 Tai TY, Wang CY, Lin LL, Lee LT, Tsai ST, Chen CJ. A case-control study on risk factors for type 1 diabetes in Taipei city. Diabetes Res Clin Pract 1998;42:197-203.

9 Jones ME, Swerdlow AJ, Gill LE, Goldacre MJ. Pre-natal and early life risk factors for childhood onset diabetes mellitus: a record linkage study. Int J Epidemiol 1998:27:444-9.

10 McKinney PA, Parslow R, Gurney KA, Law GR, Bodansky HJ, Williams R. Perinatal and neonatal determinants of childhood type 1 diabetes: a case-control study in Yorkshire, UK. Diabetes Care 1999;22:928-32.

11 Dahlquist G, Patterson C, Stoltesz G. Perinatal risk factors for childhood type 1 diabetes in Europe: the EURODIAB substudy 2 study group. Diabetes Care 1999:22:1698-1702.

12 Bache I, Bock T, Vølund A, Buschard K. Previous maternal abortion, longer gestation, and younger maternal age decease the risk of type 1 diabetes among male offspring. Diabetes Care 1999;22:1063-5.

13 Bingley PJ, Douek IF, Rogers CA, Gale EAM. Influence of maternal age at delivery and birth order on risk of type 1 diabetes in childhood: prospec tive population based family study. BMJ 2000;321:420-4.

14 Byrnes G. Flawed analysis invalidates conclusions. http://bmj.com/cgi/ eletters/321/7258/420\#EL2 (accessed 12.08.2000).

15 EURODIAB ACE Study Group. Variation and trends in incidence of childhood diabetes in Europe. Lancet 2000;355:873-6.

16 Preston DL, Lubin JH, Pierce DA, McConney ME. Epicure user's guide. Seattle: Hirosoft International Corporation, 1993

17 Stene LC, Magnus P, Lie RT, Søvik O, Joner G, the Norwegian Childhood Diabetes Study Group. Birth weight and childhood onset type 1 diabetes: population based cohort study. BMJ 2001;322:889-92.

18 Dahlquist G, Blom L, Lønneberg G. The Swedish childhood diabetes study - a multivariate analysis of risk determinants for diabetes in different age groups. Diabetologia 1991;34:757-62.

19 Berendes HW, Forman MR. Delayed childbearing: trends and consequences. In: Kiely M, ed. Reproductive and perinatal epidemiology. Boca Raton: CRC Press, 1991:28-41.

20 Skjærven R, Wilcox AJ, Lie RT, Irgens LM. Selective fertility and the distortion of perinatal mortality. Am J Epidemiol 1989;128:1352-63.

21 Morin-Papunen L, Tiilikainen A, Hartikainen-Sorri AL. Maternal HLA immunization during pregnancy: presence of anti HLA antibodies in half of multigravidous women. Med Biol 1984;62:323-5.

22 McKinney PA, Okasha M, Parslow RC, Law GR, Gurney KA, Williams R, et al. Early social mixing and childhood type 1 diabetes mellitus: a casecontrol study in Yorkshire, UK. Diabetic Med 2000;17:236-42.

23 Ákerblom HK, Knip M. Putative environmental factors in type 1 diabetes. Diabetes Metab Rev 1998:14:31-67.

(Accepted 16 May 2001) 